\title{
TEORÍA Y PRÁCTICA DE LA DIGNIDAD EN CUIDADOS PALIATIVOS: UNA REVISIÓN
}

\author{
THEORY AND PRAXIS OF DIGNITY IN PALLIATIVE CARE: A REVIEW
}

\author{
David Rudilla', Pilar Barreto ${ }^{2}$ y Amparo Oliver ${ }^{2}$ \\ I Servicios de Hospitalización Domiciliaria-Hospital General Universitario Valencia \\ 2 Facultad de Psicología. Universidad de Valencia.
}

Resumen

Se realiza un estudio de revisión sobre la dignidad humana, con el objetivo de entender este concepto a nivel teórico en los cuidados paliativos, y además, saber en qué medida estas ideas están desarrolladas a nivel práctico. Se realiza un estudio de textos centrados en revisiones de la dignidad humana desde diferentes disciplinas: filosofía, derecho, religión, bioética, atención sanitaria y cuidados paliativos. También se realiza un trabajo consistente en hallar coincidencias entre los diferentes puntos de vista, encontrando algunas semejanzas entre todas las disciplinas pero también diferencias que en Cuidados Paliativos generan el debate actual. Los autores consideran que es necesaria una revisión del tema de la dignidad humana más cercana a la práctica clínica en Cuidados Paliativos.

Palabras clave: Dignidad, revisión, cuidados paliativos, filosofía, derecho, bioética, atención sanitaria.

\begin{abstract}
A review study on human dignity is performed in order to theoretically understand this concept in the framework of palliative care, and in which extent these ideas have been developed on a practical level. Specifically, a review of those texts focused on human dignity from philosophy, law, religion, bioethics, health care and palliative care was carried out. This review also tried to find and report some similarities and differences among different approaches. Those differences are the basis of current debate on palliative care. Authors conclude that any approach to human dignity should be closer to the clinical practice in palliative care.
\end{abstract}

Key words: Dignity, review, palliative care, philosophy, law, bioethics, healthcare.

\section{Correspondencia:}




\section{ASPECTOS TEÓRICOS Y PRÁCTICOS SOBRE LA DIGNIDAD EN CUIDADOS PALIATIVOS}

\section{Planteamiento del problema}

El alivio del sufrimiento es el objetivo principal y básico de los cuidados paliativos. La Organización Mundial de la Salud (OMS) estimó que en el año 2002, 57 millones de personas murieron, de las cuales 7 millones lo hicieron por neoplasias malignas. Franks et al. (1) estimaron que, sólo en el Reino Unido, el 25\% de los pacientes diagnosticados de cáncer en estadío avanzado necesitarían cuidados paliativos, y el $65 \%$ cuidados paliativos en su domicilio. Conseguirlo, es dar calidad de vida al paciente. El tratamiento de síntomas físicos, emocionales, espirituales y sociales incluyendo estudios y trabajos cuya medida de efectividad es que el paciente encuentre dicho alivio. No es de extrañar que en todos estos estudios, la preservación de la dignidad se exprese de una manera clara y meridiana. Así pues, el creciente interés que la calidad de vida ha ido tomando en el área de los cuidados paliativos supone un crecimiento del estudio de la dignidad.

La dignidad es un concepto fundamental en la ética clínica (Chochinov, 2002(2); Lothian y Philip, 2001(3); Pannuti y Tanneberger, 1993(4); Pullman, 1999, $2002^{(5,6)}$ ) y la práctica profesional (Jacobs, $200{ }^{(7)}, 2001^{(8)}$; Mairis, 1994 ${ }^{(9)}$; Priester, 1992 ${ }^{(10)}$; Seedhouse y Gallagher, 2002 ${ }^{(11)}$; Soderberg, Gilje y Norberg, $\left.1997^{(12)}\right)$, así como en la bioética y el bioderecho internacionales (Andorno, 2009 ${ }^{(13)}$; Beyleveld y Brownsword, 2001(14); Gurnham, 2005(15); Petermann, 1996(16)). Richard Horton, editor de The Lancet, sostiene que la dignidad debe ubicarse como principio rector del movimiento de salud mundial (Horton, $\left.2004^{(17)}\right)$. Los promotores de la salud y los derechos humanos señalan que la dignidad puede ser un vínculo que explique la relación entre la promoción y protección de los derechos humanos y la situación sanitaria. El Ilamado de las Naciones Unidas en favor de un "derecho a la salud" supone una relación recíproca entre la salud y la dignidad cuando describe la salud como requisito indispensable para "vivir dignamente" (Observación general núm. $14,2000)^{(18)}$.

Debates precisamente sobre la "muerte digna" Ilenan con frecuencia todo tipo de literatura científica o divulgativa. Sin embargo parece que el debate está continuamente abierto, y en gran medida se debe a la complejidad del concepto en sí dada su importancia en cuanto a las implicaciones prácticas que supone el contenido teórico sobre el que se trabaje. ¿Por qué, si tenemos claro el objetivo de los cuidados paliativos, no está claramente definida la dignidad en esta actividad asistencial? Quizá se deba a que hablamos de un concepto que se nutre de campos tan complejos como la filosofía, la teología, el derecho y hasta la teoría política, y que incluso desde el abordaje de la bioética, surgen conflictos de intereses por este amplio enfoque y formas. Se calcula que hay 1.200 libros y $11 \mathrm{mil}$ artículos sobre dignidad publicados en inglés desde 1970 (Witte, 2003)(19), abarcando ejemplos de trabajos teóricos y empíricos de todas las disciplinas que se han ocupado del estudio de la dignidad incluyendo muchas de las obras consideradas de gran influencia para el saber contemporáneo.

Todo este enfoque sobre la dignidad en el ámbito sanitario no se produce de manera independiente y aislada, ni cuenta con un desarrollo evolutivo propio, más bien se produce por todo el cambio que sobre el tema de la dignidad se ha ido produciendo con el paso de los años. La mayoría de los trabajos se centran en la calidad de vida y el control de síntomas, pudiéndose entender que la atención hacia estos dos constructos es la preservación de la dignidad. En 
cuidados paliativos $(\mathrm{CP})$ se habla de muerte digna como un derecho humano fundamental puesto de manifiesto en múltiples declaraciones (WHO 2007(20); European Association for Palliative Care, 2007(21)), de hecho, CP y Derechos Humanos están basados en los principios de la dignidad, de la universalidad y no discriminación (Gwyther et al. 2009)(22), pero todos ellos no se solapan con el concepto de dignidad si atendemos al desarrollo de este concepto en las diferentes disciplinas que lo abordan.

Este trabajo de revisión no pretende llevar a cabo un examen exhaustivo más bien realizar una exploración de los aspectos teóricos y sus implicaciones prácticas respecto a qué entendemos por dignidad en cuidados paliativos hoy en día.

\section{Objetivos}

Este estudio con carácter exploratorio cuenta pues con los siguientes objetivos:

- Revisar las principales aproximaciones al concepto de dignidad (filosofía, derecho, religión, bioética, atención sanitaria)

- Revisar el uso del concepto de dignidad en el marco terapéutico de los Cuidados Paliativos en España

- Identificar los contenidos de cada aproximación más representativos y reconocidos por la literatura

- Encontrar coincidencias entre las diferentes aproximaciones

- Categorizar dichas coincidencias

- Identificar la presencia de dichas categorías en la revisión de la literatura sobre dignidad en Cuidados Paliativos

\section{Desarrollo del tema}

Método

En primer lugar se lleva a cabo una toma de decisiones entre los miembros del equi- po investigador para señalar las disciplinas desde las que se iba a proceder a la revisión de este concepto, decidiendo que eran imprescindible para abordar la cuestión en CP las siguientes: filosofía, derecho, religión, bioética, atención sanitaria en España y Cuidados Paliativos en España.

Los textos seleccionados para la revisión de las diferentes perspectivas fueron consensuados por los investigadores, y se seleccionaron atendiendo a recomendaciones bibliográficas de publicaciones científicas reconocidas. Se irán referenciando a medida que se desarrollen las revisiones. Los criterios de selección de los textos fueron:

- Artículos cuyo objetivo fuera el desarrollo de la dignidad en alguna de las disciplinas señaladas, con una antigüedad no superior a 10 años. Las búsquedas se realizaron en las bases de datos: Medline (21 documentos), PubMed (17 documentos), CINAHL (8 documentos), Psyclnfo (9 documentos), COCHRANE (11 documentos), Sistema para la Información sobre la Literatura Gris en Europa (10 documentos).

- Capítulos de libros que trataran la dignidad en alguna de tales disciplinas

Teniendo en cuenta los objetivos del estudio, se diseñó y utilizó una hoja de registro para cada uno de los textos, en los que se anotaba:

- contenido

- concepto: breve desarrollo del contenido del mismo

- frecuencia de aparición

Se consideró importante desde el principio tener en cuenta la definición de los conceptos fundamentales que se encontraban en cada aproximación puesto que llegado el momento de la categorización 
iba a ser importante apresar las diferencias de significado según la disciplina de que se tratara.

Para la categorización, se utilizó el juicio de expertos (tres psicólogos con experiencia y actividad clínica en clínica y salud, y en CP). Los mismos profesionales revisaron los contenidos.

\section{Análisis}

Se realiza un resumen general a partir de las revisiones desarrolladas, centrándose en los aspectos más importantes e incluyendo un apartado propio y más extenso para la dignidad en CP.

Las ideas históricas sobre la dignidad sitúan este concepto en tres ámbitos relacionales fundamentales:

- entre la humanidad y Dios

- entre el individuo y la sociedad

- entre el determinismo y la libertad

Ya en la antigüedad, Cicerón ${ }^{(23)}$ desarrolla este concepto con el sentido que conservará a lo largo de muchos siglos: como el rango que otorga al ser humano su animus o mens, el cual por ser un regalo divino, le obliga a vivir conforme a la dignidad que se le ha otorgado. En este caso, no se trata de un concepto universal y hace referencia a un status superior.

En la Biblia y durante toda la Edad Media cristiana, la dignidad se interpretó como una cualidad propia de la humanidad debido a su relación única con Dios. El ser humano estaba hecho a imagen y semejanza de Dios y, por ende, en la escala de la creación se encontraba por encima de todas las criaturas, sólo después de los ángeles (Bayertz, 1996 (24); Dales, 1977(25); Gaylin, 1984(26); Hailer y Ritschl, 1996 (27); Kristeller, 1948 ${ }^{(28)}$; Moody, 1998 $\left.{ }^{(29)}\right)$. Esta categoría especial otorgaba un valor sagrado a toda vida humana. En cambio, las ideas griegas clásicas sobre la dignidad de- rivaban de una comprensión de la sociedad como una jerarquía rígida. La dignidad se atribuía a ciertos individuos o grupos en virtud de su posición o rango en esa estructura (Johnson, 1971)(30). De este modo la idea de dignidad no se refería a un valor absoluto y sagrado, sino más bien a uno relativo y social. (Un corolario de esta definición de dignidad era la idea de que cierto comportamiento se "dignificaba" por su relación con la nobleza o un alto rango.) En el Renacimiento, la famosa Oración por la dignidad del hombre de Giovanni Pico della Mirandola ubicó la dignidad en la capacidad del ser humano de ejercer la voluntad y la elección (Kristeller, 1948) ${ }^{(28)}$ : "a fin de que puedas, en la medida de tu deseo y de tu juicio, tener y poseer aquella morada, aquella forma y aquellas funciones que a ti mismo te plazcan" (Pico, 1948 [1468], p. 224) $)^{(31)}$

Por otra parte, el concepto de dignidad de Immanuel Kant integraba varias de estas primeras ideas. Escribió sobre la dignidad como una cualidad perteneciente a todos los seres humanos sensibles por su capacidad para la racionalidad y la libertad moral (Dillon, 1995 $5^{(32)}$; Gaylin, 1984(33); Meyer, $\left.1987^{(34)}\right)$. Esta noción de dignidad conllevaba la obligación de que los seres humanos recibieran un trato respetuoso, como se refleja en la conocida máxima kantiana de que se debe ver a las personas como un fin en sí mismas y nunca sólo como un medio. Partiendo de Kant, filósofos políticos como Edmund Burke, Thomas Hobbes, Mary Wollonstonecraft, Jeremy Bentham y John Stewart Mill hicieron uso de la idea de dignidad (Horton, 2004 ${ }^{(17)}$; Meyer, 1987(34); Moody, 1998 $\left.{ }^{(29)}\right)$. Aunque estos escritores diferían en cierta medida en sus aproximaciones, lograron que la dignidad dejará de entenderse como un concepto exclusivamente religioso o filosófico y se empezara a considerar un concepto con múltiples significados e implicaciones sociopolíticos (Bayertz, 1996) ${ }^{(24)}$. 
Así mismo, en esta revisión, se ha encontrado que la dignidad se ha descrito tanto como un fenómeno objetivo, como uno subjetivo, como público y como privado (Meyer, 1989) ${ }^{(34)}$, individual y colectivo (Andorno, 2009) ${ }^{(13)}$, interno e intrínseco y externo y extrínseco (Jacelon, 2003) ${ }^{(35)}$, como jerárquico y democrático (Dillon, 1995) (32), incondicional y estático y contingente y dinámico; como inherente, conferido o conseguido (Dales, 1977) ${ }^{(25)}$; como descriptivo y preceptivo (Kolnai, 1995) ${ }^{(36)}$. Es evidente que a partir de tal confusión conceptual en el lenguaje encontremos denominaciones que se refieren a dignidad básica, dignidad humana, dignidad social y dignidad personal. Quizá, dada la complejidad y los distintos componentes tematizados se podría pensar en la dignidad como un mero recurso retórico impreciso y estereotipado, totalmente subjetivo y que lleva a una continua contradicción. Sin embargo, tras la reflexión, se considera que el conjunto de los significados de la dignidad podrían agruparse en torno a dos significados: dignidad humana y dignidad social

- Dignidad humana: En tanto que es un valor inherente e inalienable que pertenece a todo ser humano por el solo hecho de ser un humano. Poseen dignidad humana la especie, los colectivos (grupos o pueblos) y los individuos

- Dignidad social: La dignidad social tiene como fundamento la dignidad humana y es una consecuencia de su reconocimiento. Se experimenta, otorga o gana mediante la interacción en escenarios sociales.

Sin embargo también se considera que estos dos significados son excesivamente amplios y resumen únicamente algunas partes fragmentadas de las aproximaciones sobre dignidad que desde los distintos campos se han analizado en este trabajo.

\section{Dignidad en Cuidados Paliativos}

La dignidad en CP acaba siendo el objetivo primero y último de las intervenciones a llevar a cabo. En términos generales, las investigaciones tratan el abordaje del conjunto de síntomas, persiguiendo la calidad de vida, que, como comentamos anteriormente, es el concepto más amplio en relación con la idea de muerte digna. Sin embargo, aunque existe reglamentación mediante una legislación específica en los planes de salud de nuestro país la atención al final de la vida, diferentes revisiones que se llevan a cabo a nivel mundial ofrecen una visión de lo que está ocurriendo en nuestro país: se concluye por ejemplo que existe un vacío entre la investigación y la práctica en CP (Bethesda, 2013) $)^{(37)}$. En este sentido resulta interesante señalar que el primer estudio realizado en Holanda sobre la eutanasia y otras decisiones médicas en el final de la vida (Medical Decisions to End Life, MDEL) se llevó a cabo con profesionales médicos (un total de 405) que habían atendido 7000 fallecimientos. Según estos profesionales "la pérdida de la dignidad" fue la razón mayoritaria a la base del deseo de morir por parte del paciente (un 57\% de los casos). Otras razones se referían al dolor (un 5\%), dolor como parte de una constelación de síntomas $(46 \%)$ y finalmente, sentirse dependiente de otros (un 33\%) (Van Der Maas, Van Delden, Pijnenbirg, Looman, 1991) $)^{(38)}$.

¿En qué medida podemos asegurar que en Cuidados Paliativos el objetivo de la dignidad está operativizado?

El progreso técnico-médico ha supuesto una gran mejora de las condiciones de vida de la persona, pero al mismo tiempo, con el recurso a una complicada técnica en el tratamiento de las distintas enfermedades, se ha incrementado el número de enfermos que requieren cuidados especiales en los últimos días de su vida.

En la actualidad, con frecuencia existe una deficiencia en la utilización de crite- 
rios para la toma de decisiones al final de la vida, circunstancia manifestada por los distintos especialistas que trabajan con enfermos paliativos, ancianos, o con pacientes crónicos, como pueden ser los enfermos de Alzheimer. Estas cuestiones que, anteriormente podían solucionarse con arreglo a la lex artis, son ahora difícilmente subsumibles en ella, buscándose por parte de los especialistas (médicos y personal sanitario en general), una serie de criterios de racionalidad en los que basar las decisiones que la realidad clínica diaria les demanda. Por lo tanto podríamos decir, basándonos en los objetivos de los Cuidados Paliativos, el criterio que se debería emplear para la toma de decisiones es el respeto a la dignidad del paciente. Este valor, fundamental desde cualquier perspectiva en la que nos situemos, pierde en gran medida su virtualidad configuradora en la fase final de la vida y por ende en Cuidados Paliativos, por la ambigüedad con la que es utilizado.

En síntesis, a la dignidad en Cuidados Paliativos, dos han sido los significados que se le vienen atribuyendo:

- por un lado, derecho a morir con capacidad, en la medida de lo posi- ble, de elegir el modo, momento y lugar de la propia muerte

- por otro o como condición de aplicación de aquélla, el recurso a los cuidados paliativos con el fin de conseguir una muerte natural, sin sufrimiento (y como sufrimiento hablamos de más síntomas que el dolor).

La Sociedad Española de Cuidados Paliativos (SECPAL) ${ }^{(39)}$ publicó una Guía de Cuidados Paliativos donde establece como una de las bases de la terapéutica "La promoción de la autonomía y la dignidad del enfermo tienen que regir en las decisiones terapéuticas. Este principio sólo será posible si se elaboran 'con' el enfermo los objetivos terapéuticos."

Finalmente, el Modelo sobre la Dignidad desarrollado por el Dr. Chochinov ha sido el intento más ambicioso de entender la dignidad en Cuidados Paliativos. Chochinov et al. (2004)(40) Ilega a afirmar que, a pesar de que todos alardeamos de unos cuidados que preservan la dignidad al final de la vida, pocos centros especifican el mantenimiento de la dignidad como objetivo concreto de unos cuidados

\section{Tabla 1. Inventario de categorías principales de la dignidad, según Chochinov et al. (2002)(2)}

\begin{tabular}{|c|c|c|}
\hline $\begin{array}{c}\text { Preocupaciones relacionadas } \\
\text { con la enfermedad }\end{array}$ & $\begin{array}{l}\text { Preservación de la dignidad } \\
\text { (personal) }\end{array}$ & $\begin{array}{l}\text { Preservación de la dignidad } \\
\text { (social) }\end{array}$ \\
\hline $\begin{array}{l}\text { Nivel de independencia } \\
\text { - } \text { agudeza cognitiva } \\
\text { - capacidad funcional } \\
\text { Síntomas de malestar } \\
\text { - malestar físico } \\
-\quad \text { malestar psicológico }\end{array}$ & $\begin{array}{l}\text { Perspectivas que } \\
\text { conservan la dignidad } \\
-\quad \text { continuidad del yo } \\
-\quad \text { conservar los roles } \\
-\quad \text { dejar un legado } \\
-\quad \text { mantener el orgullo } \\
-\quad \text { la esperanza } \\
-\quad \text { autonomía / control } \\
-\quad \text { aceptación } \\
-\quad \text { resistencia / espíritu de } \\
\text { lucha }\end{array}$ & $\begin{array}{l}\text { Esfera de privacidad } \\
-\quad \text { Apoyo social } \\
-\quad \text { Calidad del cuidado } \\
-\quad \text { Carga de los otros } \\
\text { - } \\
\quad \text { Preocupaciones con el } \\
\quad \text { legado }\end{array}$ \\
\hline
\end{tabular}




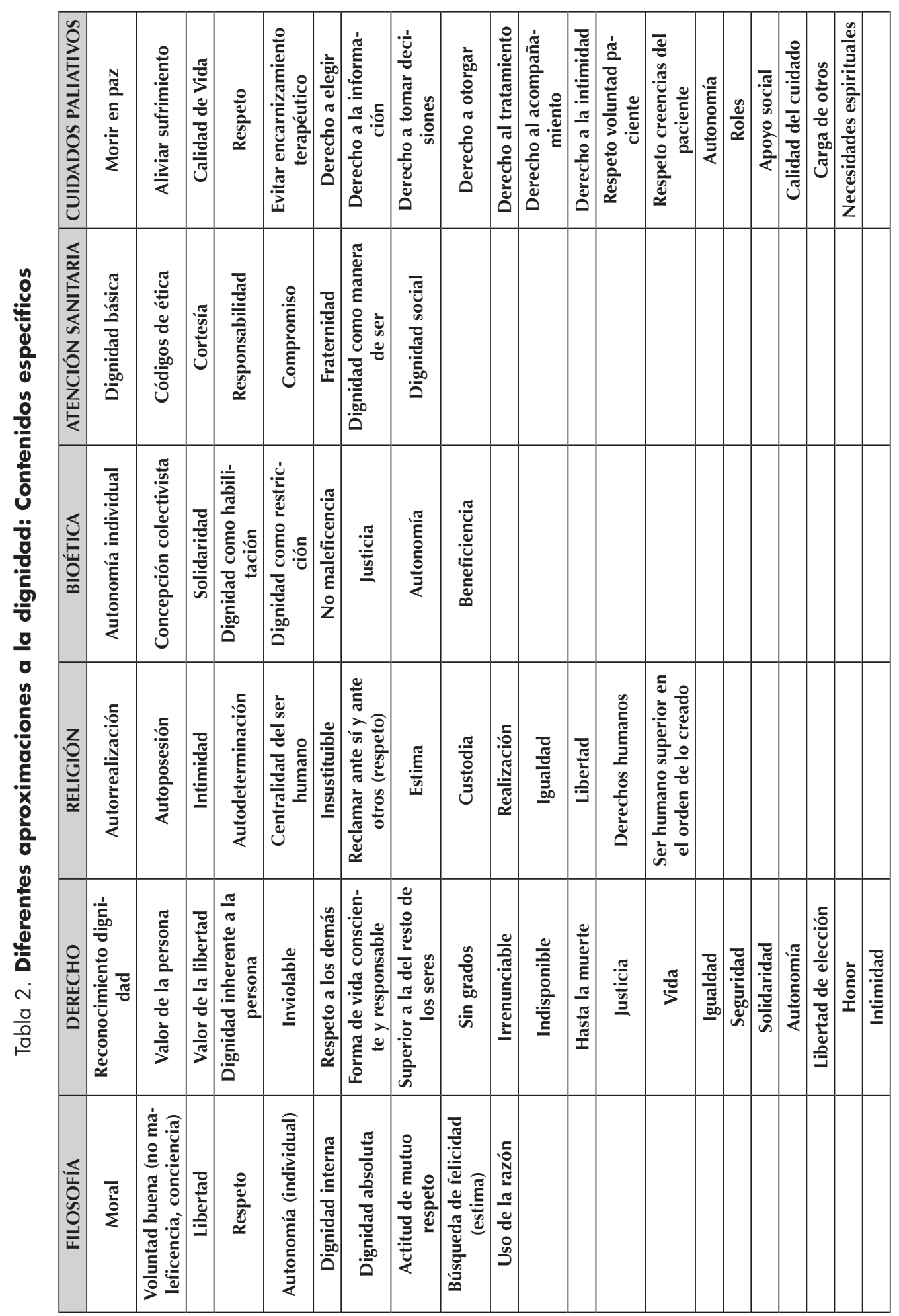


de calidad. Para subsanar esta deficiencia, y basándose en observaciones empíricas, Chochinov et al. (2002)(2) han desarrollado un modelo de dignidad en el final de la vida que proporciona un "mapa terapéuti-

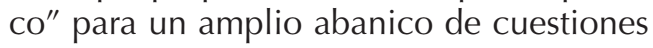
fisiológicas, psicológicas, sociales y existenciales, cuestiones todas que pueden afectar el modo como los diversos individuos perciben la dignidad ante la muerte. De estas percepciones se han extraído tres categorías básicas: (1) Preocupaciones relacionadas con la enfermedad, (2) Cuestiones que afectan la preservación de la dignidad desde el punto de vista de la persona, y (3) Cuestiones que afectan a la preservación de la dignidad desde el punto de vista social. Este modelo, ilustrado en la tabla 1 , sirve como una base para entender cómo se vive la dignidad en final de vida (Chochinov et al., 2004) ${ }^{(40)}$.

\section{RESULTADOS}

Los resultados del análisis de los artículos y textos referenciados a lo largo del desarrollo del tema, se encuentran en la tabla 2.

Atendiendo a estos resultados, en un inicio se categorizó la información agrupando los contenidos de las distintas disciplinas que se encontraron similares en función de la descripción del concepto revisado. Para esto se realizaron sesiones de discusión entre los tres psicólogos sobre las correspondencias entre los significados, revisando cuando se consideraba necesario los textos de dónde se habían extraído dichos significados. Se optó por identificar qué concepto era común en todas las disciplinas, puesto que la propia literatura nos informa que la dignidad presenta matices importantes.

Los resultados descriptivos relativos a la coincidencia de contenidos de la dignidad en las diferentes disciplinas se muestran en la tabla 3. Los contenidos coincidentes fueron agrupados teniendo en cuenta su semejanza. Tales contenidos coinciden en gran medida con los expresados en revisiones previas como la de $\mathrm{Gracia}^{(41)}$, quien comenta que términos como "dignidad humana", "dignidad personal", "derechos humanos", están siendo muy empleados, pero en ocasiones sin una debida e intensa valoración del ser humano.

Se señalaron aquellos contenidos que se encontraron en todas las aproximaciones revisadas:

- Valor de la persona

- Respeto de los demás (beneficencia, cortesía)

- Vida

- Igualdad

- Solidaridad

Por otro lado también se señaló aqueIlas que coincidieron en la casi totalidad, menos en una:

- Voluntad buena (no maleficencia, conciencia)

- Autonomía (individual)

- Dignidad interna

- Dignidad absoluta

- Actitud de mutuo respeto

- Seguridad

- Intimidad

En cuanto a los conceptos que se encontraron respecto a la dignidad en cuidados paliativos fueron la casi totalidad de los expuestos anteriormente

- Valor de la persona

- Respeto de los demás (beneficencia, cortesía)

- Vida

- Igualdad

- Solidaridad

- Voluntad buena (no maleficencia, conciencia)

- Autonomía (individual) 


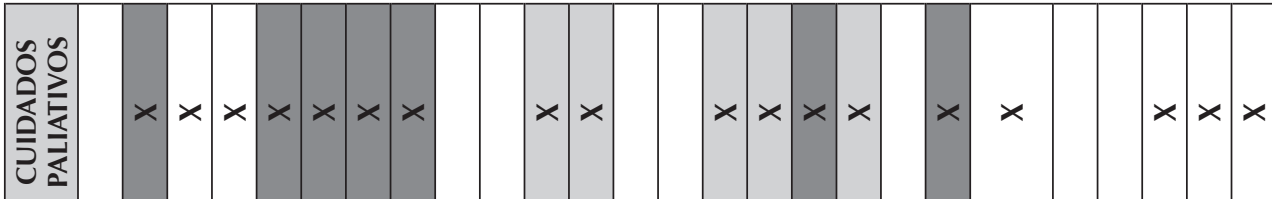

Z

을

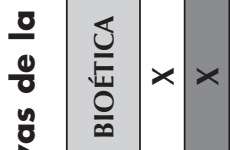

$\underset{\frac{\mathrm{O}}{\mathrm{U}}}{\frac{\mathrm{O}}{\underline{\underline{u}}}} \mid \times \times \times \times \times$

$\times \quad x \times x \quad x \times$

$\times \times \times$



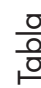

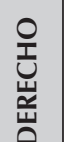

$x \times x \times x \quad x \times x \times x \times x \times x$

$\times$

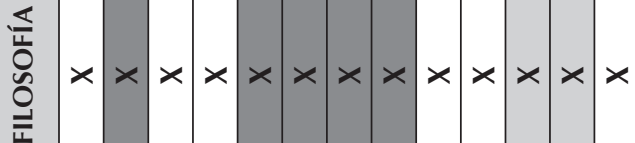

$x \times \times$

$\times$

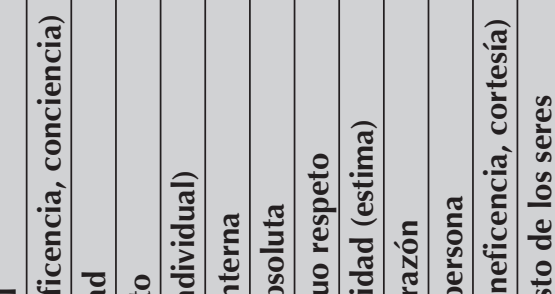

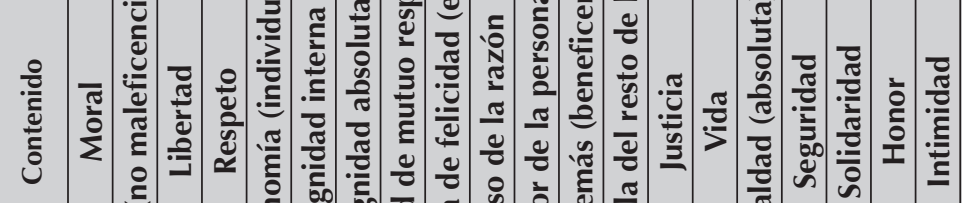

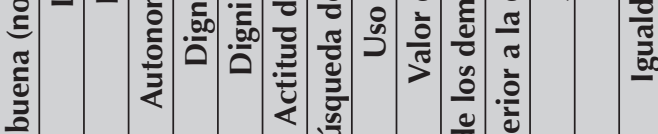

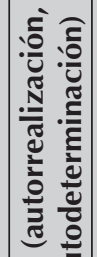

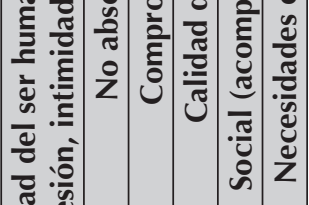

를

吾

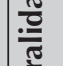

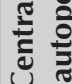


- Dignidad interna

- Dignidad absoluta

- Actitud de mutuo respeto

- Seguridad

- Intimidad

- Libertad *

- Respeto *

- Calidad de Vida *

- Social *

- Necesidades espirituales *

Los conceptos señalados con asterisco se encontraron fundamentalmente en Cuidados Paliativos aunque también en alguna otra disciplina.

\section{DISCUSIÓN Y CONCLUSIONES}

Evidentemente la dignidad cuenta con un cuerpo amplio y extenso, como comentábamos en la introducción, pero llama la atención que tal variabilidad, si bien es coincidente en muchas de las disciplinas, la gran amplitud de contenido puede dificultar la generalización en la investigación ya que puede enmascarar conceptos que podrían ser determinantes. No es de extrañar pues que la dignidad siga siendo un concepto diana de análisis y que en función del abordaje, produzca variedad de resultados y genere debates a todos los niveles.

La dignidad en cuidados paliativos se nutre de las aproximaciones que hemos detallado a lo largo del trabajo, y conduce a intentar conciliar algunos conceptos que pueden estar en una de las fuentes como el derecho frente a otros que no se contemplan en la aproximación bioética. También conceptos como respeto van recibiendo matices que amplían o reducen el significado sobre manera.

Hemos nombrado el modelo de la dignidad desarrollado por el Dr Chochinov y en la literatura científica existen cada vez más trabajos que refuerzan el beneficio de la Terapia de la Dignidad basada en dicho modelo. No obstante, en este modelo que ha sido analizado en este estudio, no figuran una serie de conceptos que sí que se consideran importantes tanto en otras disciplinas como en otras aproximaciones dentro de los cuidados paliativos; o también aproximaciones más reducidas en cuanto a la idea de dignidad dentro de los cuidados paliativos. Nos referimos a la bondad, a la igualdad, a la solidaridad y a la actitud de respeto mutuo, entre las que se han encontrado como más coincidentes. Esto viene a indicarnos que sobre un denominador común, la especificidad de cada persona prima "su dignidad".

Las autoridades políticas y sanitarias han puesto en marcha planes estratégicos en España que aseguren unos Cuidados Paliativos de calidad a todos los ciudadanos, aunque queda claro que "la dignidad" que se pretende preservar puede que no se ajuste a la que realmente se precise, precisamente por lo complejo de conjugar todas las disciplinas, siendo las más complejas la religión y el derecho.

¿Podemos afirmar entonces que la dignidad está asegurada en los cuidados paliativos? La respuesta parece ser que no del todo, por lo que sería muy interesante plantearnos qué debemos hacer para aprehender la necesidad de la dignidad en nuestros pacientes paliativos, en sus familiares y en los profesionales que los atienden. Es decir, tal vez debamos realizar un trabajo consecuente con lo puesto de manifiesto en este estudio: en función de lo que entienden por dignidad los pacientes paliativos, los familiares y los profesionales, asegurar que se cubren estas necesidades.

\section{REFERENCIAS BIBLIOGRÁFICAS}

1. Franks PJ, Salisbury CX, Bosanquet N, et al. The level of need for palliative care: A systematic review of the literature. Palliat Med 2000;14:93-104. Doi: $10.1191 / 026921600669997774$ 
2. Chochinov HM, Hack T, McClement S, Kristjanson L, Harlos M. Dignity in the terminally ill: A developing empirical model. Soc Sci Med 2002;54:433-43. Doi: 10.1016/S0277-9536(01)00084-3

3. Lothian K, Philip I. Maintaining the dignity and autonomy of older people in the healthcare setting. British Med J 2001;322:668-70.

4. Pannuti F, Tanneberger S. Human Dignity: A Human becoming ethical phenomenon. J Nurs Sci 2010;23:257-62.

5. Pullman D. The ethics of autonomy and dignity in long-term care. Can J Aging 1999;18;26-49. Doi: 10.1017/ S0714980800009612

6. Pullman D. Human dignity and the ethics and aesthetics of pain and suffering. Theoretical medicine and bioethics 2002;23:7594. Doi: 10.1023/A:1019521923979

7. Jacobs B.B. Respect for human dignity in nursing: Philosophical and practical perspectives. Can J Nurs Res 2000; 32:15-33.

8. Jacobs B.B. Respect for human dignity: a central phenomenon to philosophically unite nursing theory and practice through consilience of knowledge. Adv Nurs Sci 2001;24:17-35. Doi: 10.1097/00012272200109000-00005

9. Mairis E.D. Concept clarification in professional practice dignity. J Adv Nurs 1994;19;947-53.

10. Priester R. A values framework for health system reform. Health Aff (Millwood) 1992;11:84-107. Doi: 10.1377/hlthaff.11.1.84

11. Seedhouse D, Gallagher A. Undignifying situations. J Med Ethics 2002;28:368-72. Doi: 10.1136/jme.28.6.368

12. Söderberg A, Gilje F, Norberg A. Dignity in situations of ethical difficulty in intensive care. Intensive Crit Care Nurs 1997;13:135-44. Doi: 10.1016/S09643397(97)80857-9

13. Andorno R. Human dignity and human rights as a common ground for a global bioethics. J Med and Philos 2009;34:22340. Doi: 10.1093/jmp/jhp023

14. Beyleveld D, Brownsword R. Human Dignity in Bioethics and Biolaw. Oxford: Oxford University Press; 2001.

15. Gurnham D. The mysteries of human dignity and the brave new world of human cloning. Social Legal Studies 2005:14;197.

16. Petermann T. Human dignity and genetic tests. Sanctity of life and human dignity. Kluwer Academic Publishers, 1996. p.123-38.

17. Horton R. Rediscovering human dignity. J Lancet 2004;364:1081-5. Doi: 10.1016/ S0140-6736(04)17065-7

18. Organización Mundial de la Salud. Mejorar el desempeño de los sistemas de salud. Informe sobre la salud en el mundo. Ginebra: OMS; 2000.

19. Witte jr. J. Between sanctity and depravity: Human dignity in protestant perspective. In: Kraynak RP, Tinder G, editor. In defense of human dignity. Essays for our times. Notre Dame, 2003. p.119-38.

20. Organización Mundial de la Salud. En línea. [actualizada 17 febrero 2014; consultado 14 febrero 2014]. Disponible en: http://www.who.int/

21. European Association for Palliative Care. En línea [actualizada 17 febrero 2014; consultado 14 febrero 2014]. Disponible en: http://www.eapcnet.eu/

22. Gwyther I, Brennan F. Adavancing palliative care as a human right. En: Ponencia: $17^{\text {th }}$ Congress on Palliative. Montreal; QC; 2008.

23. Cicerón, M T. Sobre los deberes. Madrid: Alianza, 1989.

24. Bayertz K. Human dignity: Philosophical origin and scientific erosion of an idea. In: Bayertz K, editors. Sanctity of life and human dignity. Dordrecht, Kluwer Academic Publishers; 1996. p.73-90.

25. Dales RC. A medieval view of human dignity. J Hist Ideas 1977;38:557-72. Doi: $10.2307 / 2708687$

26. Gaylin W. In defense of the dignity of being human. Hastings Center Report 1984;14:18-22. Doi: 10.2307/3561161 
27. Hailer M, Ritschl D. The general notion of human dignity and the specific arguments in medical ethics. In: Bayertz K, editor. Sanctity of life and human dignity. Dordrecht, Kluwer Academic Publishers, 1996. p. 91-106.

28. Kristeller PO. Introduction. In: Cassirer E, Kristkeller PO, Randall Jr, editor. The renaissance philosophy of man. Chicago, University of Chicago Press, 1948. p. 215-22.

29. Moody HR. Why dignity in old age matters. J Georontol Soc Work 1998;29;2636. Doi: 10.1300/J083V29N02_02

30. Johnson JP: Human dignity and the nature of society. The Philosophy Forum $1971 ; 10: 213-31$.

31. Pico, G.D.M. Discurso sobre la dignidad del hombre, Traducción de Adolfo Ruiz Díaz. México, UNAM. 2004.

32. Dillon, RS. Introduction. In: Dillon RS, editor. Dignity, character, and self-respect. New York: Routledge; 1995. p.1-49.

33. Gaylin W. In defense of the dignity of being human. Hastings Center Report 1984;14:18-22. Doi: 10.2307/3561161
34. Meyer, M. Dignity, Rights and Self-Control. Ethics 1989;99. Doi: 10.1086/293095

35. Jacelon, C.S. The dignity of elders in an acute care hospital. Qual Health Res 2003;13;543-56. Doi: 10.1086/293095

36. Kolnai A. Dignity Philosophy 1976;51;251-71.

37. Bethesda MD. The science of end-of-life and palliative care. A review of research trends and funding, 1997-2010. National Institutes of Health, National Institute of Nursing Research, 2013.

38. Van Der Maas PJ, Van Delden JJ, Pijnenbirg L, Looman CW. Euthanasia and other medical decisions concerning the end of life. J Lancet. 1991;338:669-74. Doi: 10.1016/0140-6736(91)91241-L

39. Guía de Cuidados Paliativos SECPAL. En línea. [Acceso 14 febrero 2014]. Disponible en: http://www.secpal.com/guiacp/

40. Chochinov, H.M, Hack, T, Hassard T, Kristjanson J, McClement S, Harlos, M. Dignity and psychotherapeutic considerations in end-of-life care. J Palliative Care 2004;20:134-42.

41. Gracia, D. Fundamentos de bioética. Madrid: Editorial Triacastela, 2007 